\title{
Probing different time-scales of oscillatory fluctuations in visual awareness: from behavior to phase
}

\author{
Nienke Hoogenboom ${ }^{1}$ and Vincenzo Romei ${ }^{2,3}$ \\ Centre for Cognitive Neuroimaging, Institute of Neuroscience and Psychology, College of Medical, Veterinary and Life Sciences, University of Glasgow, Glasgow, UK \\ 2 Wellcome Trust Centre for Neuroimaging at University College London, Institute of Neurology, University College London, London, UK \\ 3 Institute of Cognitive Neuroscience, University College London, London, UK \\ ${ }^{*}$ Correspondence: nienke@psy.gla.ac.uk
}

\section{A commentary on:}

To see or not to see: prestimulus alpha phase predicts visual awareness.

by Mathewson, K. E., Gratton, G., Fabiani, M., Beck, D. M., and Ro, T. (2009). J. Neurosci. 29, 2725-2732.

The phase of ongoing EEG oscillations predicts visual perception.

by Busch, N. A., Dubois, J., and VanRullen, $R$. (2009). J. Neurosci. 29, 7869-7876.

Our brain needs to access the world around us in a most efficient manner, maintaining an optimal state for gaining maximal sensory input whilst utilizing minimal resources. Even though the brain's response to the outside world can evoke or induce fluctuations in engaged neuronal populations, the brain's internal state also fluctuates over time, allowing identical events to sometimes be noticed while on other occasions these events remain unnoticed. Ongoing rhythmical fluctuations have been proposed to account for the trial-by-trial variability in evoked responses to identical stimuli representing a non-random function of network fluctuations (Sadaghiani et al., 2010).

The role of alpha oscillations $(8-12 \mathrm{~Hz})$ in visual awareness has shifted over time. In the past, these oscillations were mainly described in the context of an idling hypothesis. Because of the seemingly passive states alpha oscillations were involved in, studying these oscillations became less attractive. Recent findings however have subscribed challenging and increasingly active roles to alpha oscillations with respect to neuronal operations.

Interestingly, alpha oscillations seem to synchronize with various states of awareness, yet on very different time-scales. The slowest time-scale involves very slow EEG alpha-amplitude envelope changes in the order of tens of seconds. These changes co- vary with spontaneous changes in the fMRIBOLD signal at a frequency of $0.1 \mathrm{~Hz}$ and might be responsible for slow modulations in visual perception (Goldman et al., 2002). The second scale involves oscillatory fluctuations in power on a sub-second time-scale. Alpha power in parieto-occipital areas occurring a few hundred milliseconds prior to stimulus presentation has been shown to predict the perception of forthcoming visual events (van Dijk et al., 2008) and to index momentary levels of cortical excitability (Romei et al., 2008). The third time-scale, in the order of milliseconds, involves the very cyclical fluctuations that define the oscillations. Two recent papers, whose findings nicely concur, have shed new light on the involvement of oscillatory phase in relation to visual awareness (Busch et al., 2009; Mathewson et al., 2009).

Both in a visual near-threshold detection paradigm (Busch et al., 2009) as well as a metacontrast masking paradigm (Mathewson et al., 2009), the phase of the ongoing oscillations prior to the moment of target presentation could predict whether or not the subsequent stimulus would be noticed. Trial-by-trial fluctuations in the neuronal response therefore modulate subsequent behavior in the absence of an induced phase bias, meaning that the phase across all trials at the time of stimulus presentation was randomly distributed, even though the upcoming target could be predicted due to a fixed onset delay. In addition to the phase effect, both studies find that an increase in alpha power will lead to a decrease in stimulus detection. These studies thereby support the growing evidence for an inverse relationship between alpha power and the percept of upcoming visual events.

Results of the studies differ to some extent in the domains of time, frequency and space. Busch and colleagues found an early power effect (500 ms prior to stimulus onset) at $8.2 \mathrm{~Hz}$ and a later phase effect (120 ms prior to stimulus onset) at $7.1 \mathrm{~Hz}$, which suggests no inter-dependency between power and phase. Mathewson and colleagues on the other hand, reported on both effects in the $100 \mathrm{~ms}$ prior to target onset and centered at $10 \mathrm{~Hz}$. In space, Mathewson and colleagues reported their phase effects over $\mathrm{Pz}$ while Busch and colleagues topographically illustrated that their phase effects were maximum over frontal regions, and base their main results on extensive time-frequency analyses taken from $\mathrm{Fz}$.

Despite these differences, both reports nicely concur in showing that perceived and unperceived events are associated with opposite phase angles. The studies also draw highly overlapping conclusions how these two features of oscillations (i.e., power and phase) subserve neuronal processing. Further research should elucidate the level of inter-dependency between both power and phase of these alpha oscillations, as suggested in the "pulsed inhibition" hypothesis by Mathewson and colleagues.

The "pulsed inhibition" hypothesis proposes different time-scales on which alpha oscillations can influence sensory perception. Spontaneous fluctuations in alpha power can be suppressed by top-down control to allow for prolonged periods of excitation and ultimately for increased target detectability. On the millisecond time-scale, emphasizing the automatic nature of the oscillatory cycle, access to the environment is possible by means of the rapid alternations between cortical excitability and inhibition.

These recent findings open new frontiers for the understanding of brain oscillations in visual perception by unraveling potential mechanisms by which brain oscillations might exert their effects on visual processing at different time-scales. A new promising approach suggests that manipulating oscillatory brain activity (Thut and Miniussi, 2009) could shed new light on the functional role of oscillations and put the basis for a 
causal (versus correlational) link between brain oscillations and sensory selection.

To this extent, Romei et al. (2010) demonstrated that visual input regulation can be selectively biased by rhythmic-TMS over visual areas in a spatial and frequency specific manner. In addition, Mathewson et al. (2010) suggested that awareness of near-threshold stimuli might be manipulated by sensory entrainment to rhythmic events by inducing phase-locked peaks of sensitivity.

In conclusion, both studies presented here convincingly show the relevance of alpha-phase in determining visual awareness for the first time. They open new challenging questions that future research needs to address. Manipulation of oscillatory brain activity might play a pivotal role in unraveling the fine-grained architecture governing the complex oscillationsbehavior interdependence at very different time scales.

\section{ACKNOWLEDGMENTS}

We thank Gregor Thut, Ole Jensen, and Marie-Hélène Grosbras for enthusiastic discussion and helpful comments on previous versions of this manuscript.

\section{REFERENCES}

Busch, N. A., Dubois, J., and VanRullen, R. (2009). The phase of ongoing EEG oscillations predicts visual perception. J. Neurosci. 29, 7869-7876.

Goldman, R. I., Stern, J. M., Engel, J. Jr., and Cohen, M. S. (2002). Simultaneous EEG and fMRI of the alpha rhythm. Neuroreport 13, 2487-2492.

Mathewson, K. E., Fabiani, M., Gratton, G., Beck, D. M. and Lleras, A. (2010). Rescuing stimuli from invisibility: Inducing a momentary release from visual masking with pre-target entrainment. Cognition 115 186-191.

Mathewson, K. E., Gratton, G., Fabiani, M., Beck, D. M., and Ro, T. (2009). To see or not to see: prestimulus alpha phase predicts visual awareness. J. Neurosci. 29, 2725-2732.

Romei, V., Brodbeck, V., Michel, C., Amedi, A., PascualLeone, A., and Thut, G. (2008). Spontaneous fluctuations in posterior alpha-band EEG activity reflect variability in excitability of human visual areas. Cereb. Cortex 18, 2010-2018.
Romei, V., Gross, J., and Thut, G. (2010). On the role of prestimulus alpha rhythms over occipito-parietal areas in visual input regulation: correlation or causation? J. Neurosci. 30, 8692-8697.

Sadaghiani, S., Hesselmann, G., Friston, K. J., and Kleinschmidt, A. (2010). The relation of ongoing brain activity, evoked neural responses, and cognition. Front. Syst. Neurosci. 23, 4-20. doi: 10.3389/fnsys.2010.00020.

Thut, G., and Miniussi, C. (2009). New insights into rhythmic brain activity from TMS-EEG studies. Trends. Cogn. Sci. 13, 182-189.

van Dijk, H., Schoffelen, J. M., Oostenveld, R., and Jensen, O. (2008). Prestimulus oscillatory activity in the alpha band predicts visual discrimination ability. J. Neurosci. 28, 1816-1823.

Received: 31 March 2010; accepted: 26 August 2010; published online: 02 November 2010.

Citation: Hoogenboom N and Romei V (2010) Probing different time-scales of oscillatory fluctuations in visual awareness: from behavior to phase. Front. Hum. Neurosci. 4:178. doi: 10.3389/fnhum.2010.00178

Copyright $($ C 2010 Hoogenboom and Romei. This is an open-access article subject to an exclusive license agreement between the authors and the Frontiers Research Foundation, which permits unrestricted use, distribution, and reproduction in any medium, provided the original authors and source are credited. 